Sánchez-Miguel, P.A.; Amado, D.; Mendo, S.; Molero, P.; Leo, F.M. (2019) Validation Positive Behaviors Questionnaire in Educative Dance. Revista Internacional de Medicina y Ciencias de la $\begin{array}{llllllll}\text { Actividad Física y el Deporte vol. } 19 & \text { (75) pp. 551-564 }\end{array}$ Http://cdeporte.rediris.es/revista/revista75/artvalidacion1044.htm

DOI: $10.15366 /$ rimcafd2019.75.011

\title{
ORIGINAL
}

\section{VALIDACIÓN DEL CUESTIONARIO DE COMPORTAMIENTOS POSITIVOS EN DANZA EDUCATIVA}

\section{VALIDATION POSITIVE BEHAVIORS QUESTIONNAIRE IN EDUCATIVE DANCE}

\author{
Sánchez-Miguel, P.A. ${ }^{1}$; Amado, D. ${ }^{2}$; Mendo, S. ${ }^{2}$;olero, P. ${ }^{1}$ y Leo, F.M. ${ }^{1}$ \\ 1 Doctor en Ciencias de la Actividad Física y del Deporte. Docente e investigador en el \\ Departamento de Didáctica de la Expresión Musical, Plástica y Corporal. Facultad de Formación \\ del Profesorado. Universidad de Extremadura (España) pesanchezm@unex.es, \\ diamal@unex.es, pmolero@unex.es, franmilema@unex.es \\ 2 Doctora en Ciencias del Deporte. Docente en el Departamento de Ciencias de la Educación, \\ Lenguaje, Cultura y Artes, Ciencias Histórico-Jurídicas y Humanísticas y Lenguas Modernas. \\ Facultad de Ciencias Sociales y Jurídicas. Universidad Rey Juan Carlos (España). \\ diana.amado@urjc.es \\ ${ }^{3}$ Doctor en Psicología. Investigador en el Departamento de Psicología y Antropología. Facultad \\ de Formación del Profesorado. Universidad de Extremadura (España) smendo@unex.es
}

Código UNESCO/UNESCO code: 6105.09 Validez de test Clasificación Consejo de Europa/Council of Europe classification: 17 Otras: Expresión Corporal

Recibido 18 de septiembre 2017 Received September 18, 2017 Aceptado 22 de abril de 2018 Accepted April 22, 2018

\section{RESUMEN}

El objetivo de la presente investigación era adaptar y validar al contexto de la danza educativa, el Cuestionario de Comportamientos Positivos en Educación Física. La muestra estuvo formada por 921 alumnos, de sexo femenino $(n=421)$ y masculino $(n=500)$, con edades comprendidas entre los 11 y los 17 años, pertenecientes a cuatro escuelas en México. Esta investigación estaba organizada por ConArte (Consorcio Internacional de Arte y Escuela). Los resultados obtenidos mostraron que el instrumento presentaba una adecuada validez factorial y consistencia interna. Asimismo, el instrumento se mostró invariante en cuanto al género y la edad de los participantes. De esta manera, este trabajo aporta evidencias científicas de que el cuestionario de comportamientos positivos en la danza educativa se muestra válido y fiable para analizar dichos comportamientos durante este tipo de clases. 
PALABRAS CLAVES: danza, propiedades psicométricas, comportamientos positivos, educación.

\begin{abstract}
The aim of the current research was to validate into the educative dance in Mexican secondary school, the questionnaire of positive behaviors in physical education. The sample was formed by 921 students, both female $(n=421)$ and male $(n=500)$ participants, ranging in age from 11 to 17 years old, belonged to four schools from Mexico. This research was organized by ConArte (International Consortium of Art and School). The results showed that the instrument had an adequate factorial validity and internal consistency.

Furthermore, the instrument was invariant regarding individuals' gender and age. Thus, this work gives empiric evidences that the questionnaire of positive behaviors in educative dance is valid and reliable to assess these behaviors.
\end{abstract}

KEYWORDS: dance, psychometrics properties, positive behaviors, education.

\title{
INTRODUCCIÓN
}

Durante mucho tiempo, la danza ha sido una actividad física y social de gran importancia (Quested y Duda, 2010). Por ello, la danza puede tener un potencial considerable para modificar las relaciones sociales y comportamientos de la gente que lo practica (Zander, Kreutzmann, West, Mettke y Hannover, 2014). En muchos países, sobre todo en Reino Unido (Dance in Schools Iniciative), Estados Unidos (Hampshire Dance, ArtsConnection), Alemania (TanzZeit), México (ConArte) se desarrollan proyectos en el contexto educativo con el objetivo de fomentar las experiencias y beneficios que la danza conlleva (Quested y Duda, 2010). En el Reino Unido, por ejemplo, la danza es una actividad muy popular con más de 5 millones de participantes cada año (DanceUK, 2015), más de 17000 jóvenes estudiando danza en centros educativos, dando empleo a más de 30000 personas (DanceUK, 2015), que están incluidos en una actividad creativa, física, expresiva... donde los participantes son inter-independientes, y aprenden a coordinar sus acciones conjuntamente.

A pesar de la poca importancia que le conceden algunos países en su promoción, es indudable la cantidad de beneficios que una práctica rigurosa, sistematizada y continua de la danza tiene sobre los alumnos, sobre todo su mejora en aspectos psicosociales como la motivación (Amado, Sánchez-Miguel, González-Ponce, Pulido-González y Del Villar, 2016), concentración, autonomía, fomento de la cooperación (Roseth, Johnson y Johnson, 2008), solidaridad, respeto y tolerancia (Lakes et al., 2016) fundamentalmente en un país como México, dónde los niveles de comportamientos antisociales y violentos son altos (Juárez, Villatoro, Gutiérrez, Fleiz y Medina-Mora, 2003), además de todos los beneficios que sobre la salud física promueve (Quin, Frazer y Redding, 2010). 
En este sentido, el contexto escolar es un medio generador de valores y comportamientos adaptativos, sin embargo, el centro educativo puede ser un contexto donde emerjan conductas agresivas y antisociales (Del Rey y Ortega, 2008; Smith, 2004), manifestándose en mayor en medida en países como México (Juárez et al. 2003), y estas conductas y comportamientos generan graves consecuencias como soledad, baja autoestima, altos valores de depresión (Smith, 2004). De esta manera, y partiendo del hecho que los comportamientos antisociales y prosociales son adquiridos (Feldman, 1989) el entorno educativo se muestra crucial para regularlos, y fomentar conductas positivas.

A diferencia de otros contextos, la danza ha sido menos estudiada desde la perspectiva científica (Quested y Duda, 2010; Winner, Goldstein y VincentLancrin, 2013), y mucho menos los estudios en el contexto educativo que han tratado de comprobar las relaciones entre la danza y los comportamientos entre los alumnos (Winner et al., 2013). Básicamente, los estudios que se han desarrollado en el contexto educativo han tratado de analizar los efectos de un programa de danza en adolescentes sobre el afecto y la capacidad de cooperación entre ellos (Zander et al., 2014) o testar el efecto producido sobre la motivación de un programa multidimensional de danza y expresión corporal en alumnos de enseñanza secundaria (Amado, Del Villar, Leo, Sánchez-Oliva, Sánchez-Miguel y García Calvo, 2014).

De esta manera, y hasta dónde se conoce, son pocos los trabajos que han utilizado el contexto educativo para analizar la relevancia de los contenidos de danza sobre comportamientos positivos y/o negativos (Amado et al.,2016). En esta línea, ningún trabajo en el contexto mexicano ha valorado la importancia que adquiere un tratamiento adecuado de danza educativa, y sus consecuencias a nivel académico, comportamental, social, o cognitivo.

En relación al análisis de comportamientos en el aula, uno de los trabajos que analizó de forma conjunta la aparición de comportamientos positivos y la motivación en educación física, fue el desarrollado por Sánchez-Oliva, Viladrich, Amado, González-Ponce y García-Calvo (2014), quiénes hallaron la importancia de los procesos motivacionales como elementos relevantes en el desarrollo de comportamientos positivos en el contexto de la educación física. Previamente, Sánchez-Oliva, Leo, Sánchez-Miguel, Amado y García-Calvo (2013) testaron un modelo causal dónde comprobaron la capacidad predictiva de las regulaciones motivacionales sobre la percepción del desarrollo de comportamientos positivos en educación física. Así, se muestra la relevancia de fomentar regulaciones intrínsecas para el mayor y mejor desarrollo de comportamientos positivos como el respecto, tolerancia, igualdad, etc.

Por todo ello, el objetivo fundamental de este trabajo es adaptar y validar un instrumento para valorar los comportamientos positivos en el contexto de la danza educativa. Asimismo, este estudio tratará de testar la capacidad invariante por género y edad del instrumento, además de conocer las relaciones empíricas con constructos derivados de la teoría de la autodeterminación (Deci y Ryan, 2000) estudiados previamente. La hipótesis que formulamos es que la adaptación a la Danza Educativa del Cuestionario de Comportamientos Positivos 
presentará una adecuada validez y fiabilidad en una muestra de adolescentes mexicanos. Por otro lado, se hipotetiza que el instrumento se mostrará invariante por género y edad, y mantendrá relaciones positivas y significativas con los constructos de la teoría de la autodeterminación.

\section{MATERIAL Y MÉTODOS}

\section{Participantes}

La investigación estaba organizada por ConArte (Consorcio Internacional de Arte y Escuela) e incluía 921 alumnos, de sexo femenino $(n=421)$ y masculino $(n=$ $500)$, con edades comprendidas entre los 11 y los 17 años $(M=13.17$ años; $D T$ $=1.12$ ), pertenecientes a cuatro escuelas de México. Todos los alumnos pertenecían al primer, segundo y tercer grado, y fueron seleccionados de acuerdo con su pertenencia a distintos grupos (A, B, C, D o E).

\section{Instrumentos}

Comportamientos positivos en danza educativa. Para valorar los comportamientos positivos en el contexto de la danza educativa, se utilizó la adaptación del Cuestionario de Comportamientos Positivos en Educación Física (CCPEF) (Sánchez-Oliva et al., 2013). La adaptación, consistió en modificar la redacción de la frase introductoria "En las clases de Educación Física..." por "En las clases de danza...". El cuestionario está compuesto por 18 items que analizan los cinco factores incluidos: Respeto a las instalaciones (4 ítems: e.g. "Respeto las instalaciones de la escuela"), valoración del esfuerzo (4 ítems: e.g. "Para tener éxito es importante trabajar duro"), tolerancia y respeto a los compañeros (4 ítems: e.g. "Soy tolerante con el comportamiento de mis compañeros/as"), autocontrol (3 ítems: e.g. "Suelo controlar mis acciones") y cooperación (3 ítems: e.g. "Me encanta participar en trabajos de grupo"). Los factores obtuvieron los siguientes valores alfa de Cronbach: respeto a las normas, instalaciones y materiales .80; tolerancia y respeto a los compañeros .73; valoración del esfuerzo .68; cooperación .75; y autocontrol .71.

Apoyo a las Necesidades Psicológicas Básicas. Con el objetivo de valorar la validez criterial, se empleó la Adaptación a la Expresión Corporal del Cuestionario de Apoyo a las Necesidades Psicológicas Básicas (Amado, Sánchez-Miguel, Leo, Sánchez-Oliva y García-Calvo, 2012). El cuestionario comenzaba con la frase introductoria "En las clases de Expresión Corporal en Educación Física, nuestro profesor/a..." seguido de 12 ítems agrupados en tres factores: Apoyo a la competencia (4 ítems: e.g. "Nos ha animado a que confiemos en nuestra capacidad para hacer bien las tareas"), apoyo a la autonomía (4 ítems: e.g. "Nos ha preguntado frecuentemente sobre nuestras preferencias con respecto a las actividades a realizar") y apoyo a las relaciones sociales (4 ítems: e.g. "Ha fomentado en todo momento las buenas relaciones entre los compañeros/as de clase"). Los factores obtuvieron los siguientes valores alfa de Cronbach: apoyo a la competencia .75; apoyo a la autonomía .72; apoyo a la relaciones sociales .75 . 
Cabe destacar que en todos los cuestionarios descritos se utiliza una escala tipo Likert con cinco opciones de respuesta, desde 1 (totalmente en desacuerdo) a 5 (totalmente de acuerdo) para analizar las diferentes variables.

\section{Procedimiento}

El estudio recibió el consentimiento del Comité Ético de la Universidad de Extremadura. Todos los participantes fueron tratados en condiciones de igualdad siguiendo la guía ética de la Asociación Americana de Psicología con respecto al consentimiento, confidencialidad y anonimato en las respuestas. Antes de llevar a cabo el estudio, todos los implicados fueron informados del proceso que se iba a desarrollar, realizando hincapié en que la participación era voluntaria y que los datos se tratarían de una manera confidencial. Asimismo, se obtuvo un consentimiento informado de los padres y los directores de los centros educativos en nombre de los alumnos menores de edad incluidos en el trabajo.

Para llevar a cabo la recogida de datos, se realizó un protocolo de actuación con el objetivo de que fuera similar durante todo el proceso. En primer lugar, el investigador principal se puso en contacto con el centro educativo para solicitar su participación en el estudio y, desde el centro se contactó con los padres de los alumnos para pedir su autorización, dado que la mayoría de los alumnos eran menores de edad. La administración de la escala se llevó a cabo en horario escolar, ofreciendo las instrucciones pertinentes a los alumnos participantes e insistiendo en el anonimato. Ésta fue completada de manera individual y en un clima adecuado, que les permitía concentrarse sin tener ningún tipo de distracción. El proceso de realización de los cuestionarios duró aproximadamente 10 minutos y el investigador principal estuvo presente en el momento en que los participantes completaban los cuestionarios, e insistió en la posibilidad de preguntar cualquier tipo de duda que apareciese durante el proceso.

\section{Análisis de los datos}

Se calcularon los estadísticos descriptivos y los valores de asimetría y curtosis de cada uno de los ítems que componen el cuestionario para comprobar la normalidad univariada de los ítems. A continuación, se llevó a cabo un análisis de las propiedades psicométricas de la versión adaptada a la danza educativa del CCPEF, utilizando el análisis factorial confirmatorio mediante el programa AMOS 21. Para testar el ajuste entre el modelo teórico planteado y la matriz de datos recogida se utilizaron diferentes índices: $X^{2} / g l$ (Chi-Square / degrees of freedom), CFI (Comparative Fit Index), TLI (Tucker Lewis Index), RMSEA (Root Mean Square Error of Approximation) y SRMR (Standardized Root Mean Residual). Mediante el cálculo de la varianza media extractada, la fiabilidad compuesta y el omega de McDonald se evaluó la consistencia interna de la adaptación del CCPEF. Además se realizaron sendos análisis invariantes por género y edad, y un análisis de validez nomológica entre los factores del CCPCD y el CANPB a través de un análisis de correlaciones bivariadas, utilizando para ello el programa SPSS 21. 


\section{RESULTADOS}

\section{Análisis descriptivo de los ítems}

El valor de la media de los ítems que componen el cuestionario oscila en torno a $4 \mathrm{y}$, por tanto, al máximo otorgado dentro de una escala likert de 5 puntos (Tabla 1). Así pues, teniendo en cuenta la distribución del cuestionario donde las puntuaciones 1 y 2 se correspondían con los ítems en sentido negativo, las puntuaciones 4 y 5 con los ítems en sentido positivo y el 3 era la puntuación transitoria entre uno y otro, esto nos indica que las puntuaciones en todos los casos tienden hacia comportamientos positivos y no a los negativos. En cuanto a las pruebas de normalidad, cabe señalar que todos los valores de asimetría y curtosis fueron inferiores a 2, lo que refleja una distribución normal univariada de los datos (Bollen y Long, 1993).

Tabla 1. Estadísticos descriptivos de los ítems del cuestionario.

\begin{tabular}{|c|c|c|c|c|c|}
\hline & & $M$ & $D T$ & As & $\mathrm{Cu}$ \\
\hline 1. & Respeto las instalaciones de la escuela & 4.14 & 1.23 & -1.36 & .75 \\
\hline 2. & Lo más importante es esforzarse al máximo & 3.92 & 1.29 & -.99 & -.14 \\
\hline 3. & Ayudo a un compañero después de una caída & 3.88 & 1.25 & -.95 & -.08 \\
\hline 4. & Suelo controlar mis acciones & 3.72 & 1.33 & -.79 & -.53 \\
\hline 5. & Me encanta participar en trabajos de grupo & 3.74 & 1.35 & -.80 & -.51 \\
\hline 6. & Para tener éxito es importante trabajar duro & 4.03 & 1.23 & -1.21 & .45 \\
\hline 7. & $\begin{array}{l}\text { Soy tolerante con el comportamiento de mis } \\
\text { compañeros/as }\end{array}$ & 3.66 & 1.33 & -.70 & -.62 \\
\hline 8. & Siempre me siento controlado/a & 3.63 & 1.30 & -.66 & -.61 \\
\hline 9. & Respeto las normas impuestas por los profesores/as & 3.88 & 1.31 & -.96 & -.22 \\
\hline 10. & Siempre intento ver por el interés de mis compañeros/as & 3.72 & 1.27 & -.77 & -.39 \\
\hline 11. & Trato de no dañar las instalaciones de mi escuela & 3.95 & 1.27 & -1.04 & .02 \\
\hline 12. & $\begin{array}{l}\text { Poner empeño es muy importante para aprender y } \\
\text { mejorar }\end{array}$ & 4.02 & 1.19 & -1.11 & .36 \\
\hline 13. & $\begin{array}{l}\text { Cuando se me acaba la paciencia, sé controlar mis } \\
\text { impulsos }\end{array}$ & 3.63 & 1.34 & -.69 & -.65 \\
\hline 14. & $\begin{array}{l}\text { Me gusta trabajar con los demás en las actividades de la } \\
\text { clase }\end{array}$ & 3.86 & 1.26 & -.95 & -.09 \\
\hline 15. & Respeto los materiales de la escuela & 4.03 & 1.23 & -1.20 & -.48 \\
\hline 16. & $\begin{array}{l}\text { Acepto a mis compañeros/as independientemente de que } \\
\text { sean diferentes a mi }\end{array}$ & 3.91 & 1.24 & -.96 & -.06 \\
\hline & $\begin{array}{l}\text { Prefiero que haya actividades colectivas, para trabajar en } \\
\text { grupo }\end{array}$ & 3.78 & 1.35 & -.83 & -.49 \\
\hline & Sé controlarme cuando algo no sale como quiero & 3.77 & 1.33 & -.84 & -.46 \\
\hline
\end{tabular}

\section{Análisis factorial confirmatorio}

Con el objetivo de corroborar la estructura factorial obtenida en el estudio de Sánchez-Oliva et al. (2013), y una vez eliminados los valores atípicos (Tests for normality and outliers, AMOS) y comprobados que se cumplen los criterios de normalidad y linealidad, se ponen a prueba con el método de máxima verosimilitud (coeficiente de Mardia $=110.93$ ) tres modelos factoriales: 1 ) un factor único; 2) cinco factores relacionados; y 3) cinco factores de primer orden y uno de segundo orden. Teniendo en cuenta que el valor de $x^{2}$ es muy sensible 
a pequeñas desviaciones del modelo hipotetizado (Jöreskog y Sörbom, 1993), se utilizaron los siguientes índices para evaluar el ajuste del modelo: $X^{2} / g l, C F l$, $T L I, S R M R$ y RMSEA. En el caso del $\mathrm{x}^{2} / \mathrm{gl}$, se consideran aceptables valores inferiores a 5 (Bentler, 1995), mientras que Hu y Bentler (1999) consideran valores aceptables del GFI y los índices incrementales (CFI, TLI) iguales o superiores a .90, considerándose excelentes valores superiores a .95. Por último, el modelo será considerado con buen ajuste si el $S R M R$ es inferior a .08 y el RMSEA inferior a .06 (Hu y Bentler, 1999).

En la Tabla 2 aparecen los valores registrados en los índices de ajuste utilizados de los tres modelos analizados. En los modelos de un factor único y de cinco factores relacionados presentan un ajuste adecuado. Sin embargo, el modelo de cinco factores de primer orden con un factor de segundo orden presentan el mejor ajuste, con los siguientes valores: $X^{2} / g I(2.267)$, CFI y TLI ( $\left.\geq .925\right)$ y RMSEA $(<.06)$.

Tabla 2. Índices de bondad de ajuste de los modelos propuestos.

\begin{tabular}{lcccccc}
\hline \multicolumn{1}{c}{ Modelos } & $\mathrm{X}^{2}$ & $X^{2} / g l$ & CFI & TLI & RMSEA & SRMR \\
\hline 1 factor único & $p<.001$ & 7.547 & .827 & .805 & .095 & .144 \\
5 factores relacionados & $p<.001$ & 3.53 & .919 & .901 & .068 & .049 \\
1 factor de 20 Orden & $p<.001$ & 2.267 & .936 & .925 & .059 & .040 \\
\hline
\end{tabular}

Nota. $X^{2} / g l=$ razón de chi-cuadrado sobre los grados de libertad; $\mathrm{CFI}=$ índice de ajuste comparativo; TLI= índice de Tucker-Lewis; RMSEA= error cuadrático medio de aproximación; $S R M R=$ raíz cuadrada media residual estandarizada.

El AFC representa una estructura factorial compuesta por 18 variables observables endógenas (los 18 ítems que componen el cuestionario), cinco variables latentes endógenas (Respeto a las instalaciones, tolerancia y respeto a los demás, autocontrol, valoración del esfuerzo y cooperación) y una variable latente exógena (comportamientos positivos). En dicho modelo, cada variable latente endógena está definida por al menos 3 variables observables endógenas, tal y como recomienda Bollen (1989). El modelo estructural es recursivo, no hay relaciones de causalidad entre las variables endógenas y los errores de medida no están correlacionados, por lo que se asume la identificación del modelo, requisito indispensable para poder dar paso a la estimación de parámetros (Varela, Abalo, Rial y Braña, 2006).

En la Figura 1 se muestran las saturaciones del factor de segundo orden sobre los factores de primer orden, las cargas factoriales de cada factor de primer orden en cada ítem y el coeficiente de correlación múltiple para cada ítem. En cuanto a las cargas factoriales del factor global sobre los factores de primer orden, en todos los casos se obtuvieron saturaciones adecuadas, donde la tolerancia y el respeto a los demás, la valoración del esfuerzo y la cooperación, alcanzaron puntuaciones superiores a .90. Por el contrario, el respeto a las instalaciones y el autocontrol mostraron puntuaciones ligeramente inferiores (.72 y .85, respectivamente). Por último, también cabe destacar como todos los factores de primer orden obtuvieron cargas factoriales adecuadas en todos sus ítems, con valores superiores a .60. 


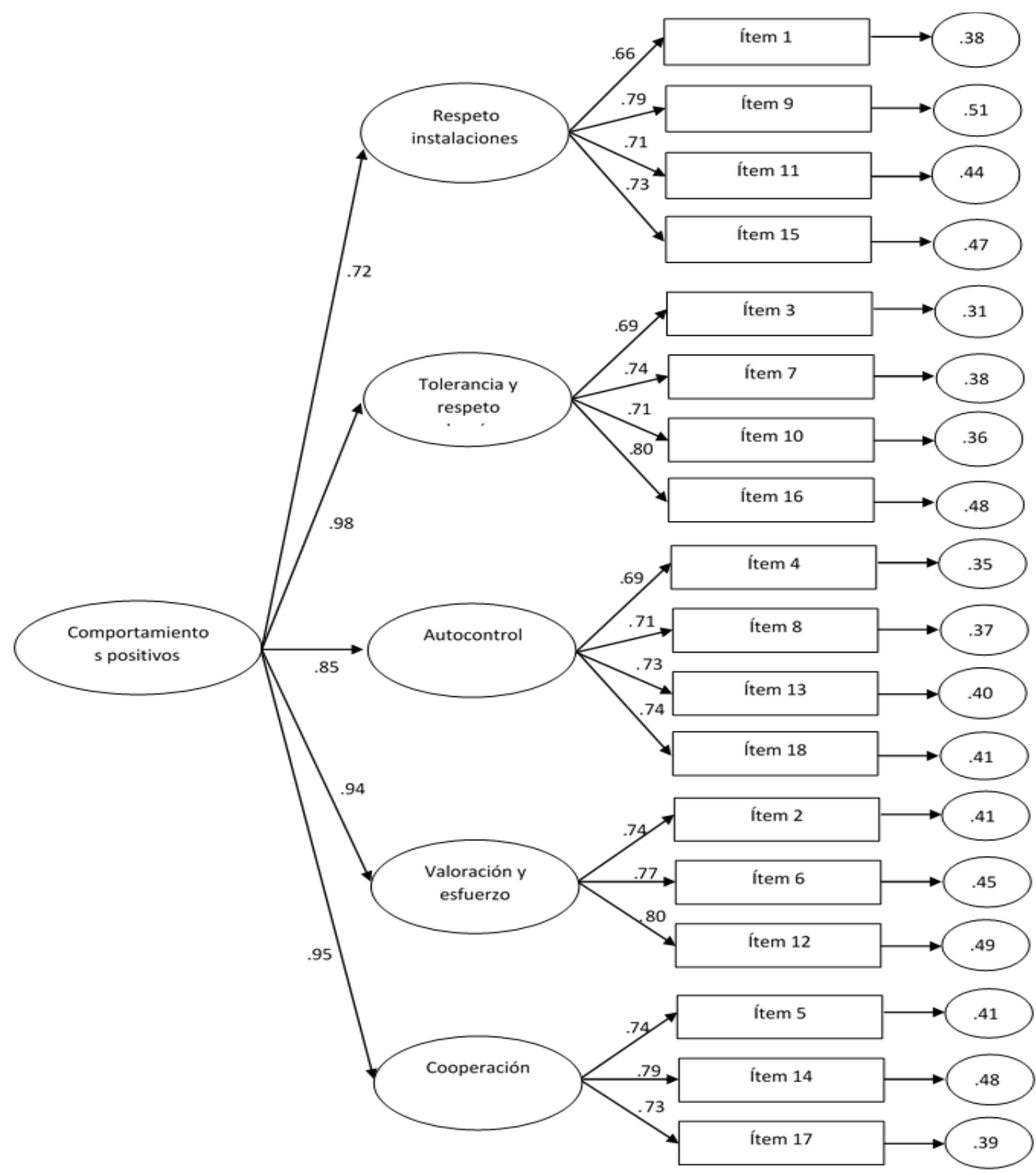

Figura 1. Modelo obtenido del análisis del estilo factorial confirmatorio.

\section{Consistencia interna}

En la Tabla 3 se presentan la varianza media extractada (VME), la fiabilidad compuesta (FC) y el omega de McDonald $(\Omega)$ de los constructos latentes. Es deseable que los valores de la VME sean iguales o superiores a $.50 \mathrm{e}$ iguales 0 superiores a .70 para la FC y el $\Omega$, por lo que se puede afirmar que el modelo de un factor único y cinco factores independientes presenta una adecuada consistencia interna. 
Tabla 3. Valores VME, FC y $\Omega$ de los constructos latentes.

\begin{tabular}{lccccc}
\hline & $\mathrm{F} 1$ & $\mathrm{~F} 2$ & $\mathrm{~F} 3$ & $\mathrm{~F} 4$ & $\mathrm{~F} 5$ \\
\hline Varianza media extractada & .508 & .509 & .504 & .593 & .568 \\
Fiabilidad compuesta & .847 & .848 & .847 & .814 & .798 \\
Omega de McDonald & .834 & .836 & .830 & 797 & 780 \\
\hline
\end{tabular}

Nota. F1=Respeto a las normas, instalaciones y materiales; F2 = Tolerancia y respeto a los compañeros; F3 = Autocontrol; F4 = Valoración del esfuerzo; F5 = Cooperación

\section{Análisis invariante por género y edad}

Para determinar si el modelo de cinco factores de primer orden y uno de segundo orden es invariante por género (190 mujeres y 170 varones), se realiza un análisis multigrupo (Tabla 4). La comparación no muestran diferencias $p<.05$ en el valor de chi cuadrado entre los diferentes modelos y los valores encontrados en el $\triangle \mathrm{CFI}$ en el modelo sin restricciones con diferencias menores de .01 de los índices CFI entre los cuatro modelos, indican que las cargas factoriales del cuestionario son equivalentes para mujeres y varones.

Asimismo, se realiza un análisis multigrupo para determinar si dicho modelo es invariante por edad (Tabla 4). Previo al análisis se establecen dos grupos de comparación en función de la edad [(grupo 1: rango 11-13 años, $n=198) ;($ grupo 2: rango 14-16 años, $\mathrm{n}=162$ )]. El análisis multigrupo no muestra diferencias $p<$ .05 en el valor de chi cuadrado entre los diferentes modelos y los valores encontrados en el $\triangle C F I$ en el modelo sin restricciones, además las diferencias entre los cuatro modelos en los índices CFI son menores de .01, indicando que las cargas factoriales del cuestionario son equivalentes entre los grupos de edad.

Tabla 4. Análisis multigrupo de invarianza por género y edad.

\begin{tabular}{ccccccccc}
\hline Modelos & $\mathrm{X} 2$ & $\mathrm{X} 2 / \mathrm{gl}$ & $\Delta \mathrm{X} 2$ & $p$ & $\mathrm{CFI}$ & $\mathrm{TLI}$ & SRMR & RMSEA \\
\hline Género & & & & & & & & \\
Modelo 1 & 811.84 & 3,007 & - & - & .930 & .918 & .060 & .047 \\
Modelo 2 & 821.32 & 2.902 & 7,638 & .730 & .931 & .924 & .064 & .044 \\
Modelo 3 & 826.18 & 2.869 & 8,162 & .707 & .934 & .930 & .064 & .042 \\
Modelo 4 & 858.91 & 2.807 & 31,140 & .102 & .924 & .923 & .070 & .045 \\
\hline Edad & & & & & & & & \\
\hline Modelo 1 & 807.07 & 2,989 & - & - & .940 & .923 & .059 & .045 \\
Modelo 2 & 818.60 & 2.882 & 8,529 & .465 & .943 & .941 & .062 & .046 \\
Modelo 3 & 828.27 & 2.876 & 16,843 & .269 & .940 & .933 & .066 & .047 \\
Modelo 4 & 845.63 & 2.763 & 38,558 & .355 & .923 & .913 & .070 & .049 \\
\hline
\end{tabular}

Nota. Modelo $1=$ Sin restricciones. Modelo $2=$ Pesos de medida. Modelo $3=$ Covarianzas estructurales. Modelo $4=$ Residuos de medida.

\section{Validez nomológica}

La validez nomológica se refiere al grado en que se pueden comprobar empíricamente las relaciones que un constructo puede mantener con otros que forman parte total o parcialmente de alguna teoría o teorías (Wilson, Spence y Kavanagh, 1989), es decir, si existe correspondencia entre la configuración teórica de los datos obtenidos y las predicciones teóricas sobre dicha configuración. 
En este caso se ha relacionado las puntuaciones del Cuestionario de Comportamientos Positivos en las clases de Danza Educativa (CCPDE), con los factores del Cuestionario de Apoyo a las Necesidades Psicológicas Básicas en las clases de Expresión Corporal (CANPB) (Amado et al., 2012) (Tabla 5). La correlación entre los factores del CCPDE y los factores del CANPB, muestra la existencia de relación directa significativas (media/baja) entre los cinco factores del CCPDE y los tres factores del CANPB (Tabla 5).

Tabla 5. Correlación de Pearson Factores del CCPDE/CANPB.

\begin{tabular}{lccccc}
\hline & Respeto & Tolerancia & Autocontrol & $\begin{array}{c}\text { Valoración del } \\
\text { Esfuerzo }\end{array}$ & Cooperación \\
\hline 1. Apoyo a la & $.338^{* *}$ & $.351^{* *}$ & $.240^{* *}$ & $.311^{* *}$ & $.295^{* *}$ \\
competencia & $.284^{* *}$ & $.326^{* *}$ & $.229^{* *}$ & $.280^{* *}$ & $.256^{* *}$ \\
2. Apoyo a la autonomía & $.325^{* *}$ & $.220^{* *}$ & $.295^{* *}$ & $.316^{* *}$ \\
\hline 3. Apoyo a las relaciones & $.329^{* *}$ & .33 & &
\end{tabular}

Nota. ${ }^{* *}$ La correlación es significativa al nivel de .01

\section{DISCUSIÓN Y CONCLUSIONES}

El objetivo principal de este trabajo era adaptar y validar un instrumento para valorar los comportamientos positivos en el contexto de la danza educativa en México. La primera hipótesis que formulamos fue que la adaptación a la Danza Educativa del Cuestionario de Comportamientos Positivos en Educación Física presentaría una adecuada validez y fiabilidad.

En primer lugar, y de acuerdo a esta hipótesis, los resultados de los análisis psicométricos y factoriales confirmatorios realizados, basado en modelos de ecuaciones estructurales mostraron una adecuada estructura factorial, consistencia interna y validez criterial del instrumento. Por consiguiente, se puede indicar que a partir de ahora este cuestionario se puede utilizar para valorar los comportamientos positivos en las clases de danza y expresión corporal.

En este sentido, se pudo comprobar que el modelo factorial es recursivo, donde no hay relaciones de causalidad entre las variables endógenas y los errores de medida no están correlacionados. Además, el análisis factorial mostró cómo los cinco factores obtuvieron saturaciones por encima de .60, indicando una adecuada validez factorial (Varela et al., 2006). Resultados similares fueron encontrados en estudios previos por otros autores (Baena-Extremera, GraneroGallegos, Bracho-Amador, Pérez-Quero, 2012; Sánchez-Oliva et al., 2013), evidenciando las propiedades adecuadas de la adaptación de instrumentos para valorar variables psicológicas. En cuanto a la fiabilidad del instrumento, se puede observar cómo todos los factores tenían unos índices Alpha de Cronbach por encima de .70 (Nunnally, 1978), lo que denota que los diferentes ítems que agrupa cada factor miden de forma similar, además de mostrar un adecuado ajuste lingüístico a la equivalencia psicológica de los constructos que se deseaban valorar, proporcionando un instrumento de medida psicológico adecuado para el contexto educativo mexicano. 
Se destaca que hasta el momento, ningún instrumento que valorase conductas y/o comportamientos se había validado y/o adaptado al contexto de la danza educativa mexicana, con los enormes beneficios que ello conlleva en un contexto donde aparecen con frecuencia conductas agresivas y antisociales (Juárez et al. 2003). La mayoría de ellos trataban de valorar comportamientos positivos en las clases de educación física, independientemente del contenido que se impartiera (Sánchez-Oliva et al., 2013), o valoraban aspectos sociales en la danza (Quested y Duda, 2010), o la satisfacción deportiva en el contexto de la educación física (Baena-Extremera et al., 2012). De esta manera, una de las aportaciones más importantes del presente trabajo es que permite valorar de forma válida y fiable los comportamientos positivos en el contexto de la danza educativa mexicana, dotando a los docentes de una herramienta útil que permita conocer la transferencia de los contenidos expresivos hacia comportamientos adaptativos.

Por otro lado, se hipotetizó que el instrumento se mostraría invariante por género y edad. Si observamos los resultados obtenidos, se muestra que los índices de carga factoriales son equivalentes para mujeres y varones, no mostrando diferencias $p<.05$ en el valor de chi cuadrado entre los diferentes modelos. De esta manera, se puede concluir que el instrumento es fiable y válido para medir comportamientos positivos, independientemente del género del alumnado. Por otro lado, el análisis multigrupo tampoco reveló diferencias $p<.05$ en el valor de chi cuadrado entre los diferentes modelos y los valores encontrados en el $\Delta C F I$ en el modelo sin restricciones, mostrando además, cargas factoriales del cuestionario equivalentes entre los grupos de edad realizados edad [(grupo 1: rango 11-13 años, $n=198$ ); (grupo 2: rango 14-16 años, $n=162$ )]. Igualmente, se concluye que el instrumento es válido y fiable, independientemente del grupo de edad del alumnado.

Estos resultados son consistentes con los hallados en trabajos previos (Sánchez-Oliva et al., 2013; Sicilia, Ferriz, Trigueros, González-Cutre, 2014) quienes hallaron invariante el modelo testado tanto en la adaptación y validación del cuestionario de apoyo a las necesidades psicológicas básicas en Educación Física como en la adaptación y validación española del Physical Activity Class Satisfaction Questionnaire (PACSQ). De esta manera, se puede observar resultados similares entre nuestro trabajo y estudios previos que han validado cuestionarios al contexto de la actividad física y el deporte.

Finalmente, y en relación a la hipótesis que planteaba que el cuestionario de comportamientos positivos en danza educativa mantendría relaciones empíricas con constructos derivados de la teoría de la autodeterminación (Deci y Ryan, 2000), se puede comprobar que esta hipótesis se confirma. En este sentido, los resultados nos muestran que los factores del cuestionario de comportamientos positivos se correlacionan de forma positiva con los factores del CANPB (Amado et al., 2012). De esta manera, se comprueba la validez nomológica del instrumento, siguiendo los postulados de la teoría de la autodeterminación (Deci y Ryan, 2000), y se observa cómo el apoyo de las necesidades psicológicas básicas, así como los diferentes tipos de regulación motivacional están asociados positivamente con comportamientos positivos (Sánchez-Oliva et al., 2013). 
Para concluir, el presente estudio ha demostrado las propiedades psicométricas del cuestionario de comportamientos positivos en la danza educativa, mostrando una adecuada validez y fiabilidad. Asimismo, el instrumento se ha mostrado invariante en cuanto al género y la edad de los participantes, lo que hace de este instrumento una herramienta útil y eficaz para aplicar en contextos de enseñanza, y valorar el grado de adquisición de comportamientos positivos por parte del alumnado.

En cuanto a las limitaciones del estudio, se destaca que la herramienta solamente se ha validado en el contexto mexicano, por lo que diferencias culturales entre países de habla hispana podría conllevar variaciones en los resultados obtenidos. Por ello, como prospectiva de futuro se establece el realizar una adaptación y validación trans-cultural del cuestionario de comportamientos positivos en danza educativa en diferentes países de habla hispana, con el objetivo de conocer las posibles similitudes y/o diferencias que pudieran existir.

\section{REFERENCIAS BIBLIOGRÁFICAS}

Amado, D., Del Villar, F., Leo, F. M., Sánchez-Oliva, D., Sánchez-Miguel, P. A. y García-Calvo, T. (2014). Effect of a multi-dimensional intervention programme on the motivation of physical education students. PLOS ONE, 9(1), e85275. doi.org/10.1371/journal.pone.0085275

Amado, D., Leo, F. M., Sánchez-Oliva, D., González-Ponce, I., Chamorro, J. L. y Pulido, J. (2012). Análisis de las propiedades psicométricas del Cuestionario de Motivación en Danza y Expresión Corporal. Actas del II Congreso Nacional de Investigación en Danza, Barcelona (España). 16 18 de Noviembre de 2012.

Amado, D., Sánchez-Miguel, P. A., Leo, F. M., Sánchez-Oliva, D. y García-Calvo, T. (2012). Adaptación a la expresión corporal del Cuestionario de Apoyo a las Necesidades Psicológicas Básicas. Electronic Journal of Research in Educational Psychology, 10(2), 867-884. doi.org/ 10.25115/ejrep.v10i27.1512

Amado, D., Sánchez-Miguel, P. A., González-Ponce, I., Pulido, J. J. y Del Villar, F. (2016). Motivation towards dance within physical education according to teaching technique and gender. South African Journal for Research in Sport, Physical Education and Recreation, 38(2), 1-16.

Baena-Extremera, A., Granero-Gallegos, A., Bracho-Amador, C. y Pérez-Quero, F. J. (2012). Spanish version of the Sport Satisfaction Instrument (SSI) adapted to physical education. Revista de Psicodidáctica, 17(2), 377-395. Doi: 10.1387/Rev.Psicodidact.4037

Byrne, B. M. (2001). Structural equation modeling with Amos: Basic concepts, applications and programming. Mahwah, NY.: Lawrence Erlbaun.

DanceUK. (2015). Dance Facts and Stats, Compiled 2015. Retrieved 2nd November 2016, from https://www.danceuk.org/resources/dance-facts/.

Deci, E. L. y Ryan, R. M. (2000). The "what" and the "why" of goal pursuits: Human needs and the self-determination of behaviour. Psychological Inquiry, 11, 227-268. doi.org/10.1207/S15327965PLI1104_01 
Del Rey, R. y Ortega, R. (2008). Bullying in poor countries: Prevalence and coexistence with other forms of violence. International Journal of Psychology and Psychological Therapy, 8(1), 39-50.

Feldman, M. P. (1989). Comportamiento criminal: Un análisis psicológico. México: Fondo de Cultura Económica.

Hu, L. y Bentler, P. M. (1999). Cutoff criteria for fit indexes in covariance structure analysis: Conventional criteria versus new alternatives. Structural Equation Modeling, 6, 1-55. doi.org/10.1080/10705519909540118

Juárez, F., Villatoro, J., Gutiérrez, M. L., Fleiz, C. y Medina-Mora, M. E. (2003). Tendencias de la conducta antisocial en estudiantes del Distrito Federal: Mediciones 1997-2003. Salud Mental, 28(3), 60-68.

Lakes, K. D., Marvin, S., Rowley, J., San Nicolas, M., Arastoo, S., Viray, L., Orozco, A. y Jurnak, F. (2016). Dancer perceptions of the cognitive, social, emotional, and physical benefits of modern styles of partnered dancing. Complementary therapies in Medicine, 26, 117-122. doi.org/10.1016/j.ctim.2016.03.007

Nunnally, J. C. (1978). Psychometric Theory. Nueva York: McGraw-Hill.

Quested, E. y Duda, J. L. (2010). Exploring the social-environmental determinants of well- and ill-being in dancers: A test of Basic Needs Theory. Journal of Sport and Exercise Psychology, 32(1), 39-60.

Quin, E., Frazer, L. y Redding, E. (2007). The health benefits of creative dance: improving children's physical and psychological wellbeing. Education and Health, 25, 31-33.

Roseth, C. J., Johnson, D. W. y Johnson, R. T. (2008). Promoting early adolescents' achievement and peer relationships: the effects of cooperative, competitive, and individualistic goal structures. Psychological Bulletin, 134, 223-246. Doi: 10.1037/0033-2909.134.2.223

Sánchez-Oliva, D., Leo, F. M., Sánchez-Miguel, P. A., Amado, D. y GarcíaCalvo, T. (2013). Desarrollo de un modelo causal para explicar los comportamientos positivos en las clases de educación física. Acción Motriz, 10, 48-58. DOI: 10.1387/RevPsicodidact.7911

Sánchez-Oliva, D., Sánchez-Miguel, P. A., Leo, F. M., Amado, D. y García-Calvo, T. (2013). Desarrollo y validación de un cuestionario para analizar la percepción de comportamientos positivos en las clases de educación física. Cultura y Educación, 25(4), 495-508.

Sánchez-Oliva, D., Viladrich, C., Amado, D., González-Ponce, I. y García-Calvo, T. (2014). Predicción de los comportamientos positivos en educación física: una perspectiva desde la teoría de la autodeterminación. Revista de Psicodidáctica, 19(2), 387-406. DOI: 10.1387/RevPsicodidact.7911

Sicilia, A., Ferriz, R., Trigueros, R. y González-Cutre, D. (2014). Adaptación y validación española del Physical Activity Class Satisfaction Questionnaire (PACSQ). Universitas Psychológicas, 13(4), 1321-1332. doi.org/10.11144/Javeriana.UPSY13-4.ayve

Smith, P. K. (2004). Bullying: recent developments. Child and Adolescent Mental Health, 9(3), 98-103. Doi: 10.1111/j.1475-3588.2004.00089.x

Varela, J., Abalo, J., Rial, A. y Braña, T. (2006). El Análisis Factorial Confirmatorio de Segundo Nivel. En J.P. Lévy y J. Varela (Eds.), 
Modelización con Estructuras de Covarianzas en Ciencias Sociales (pp.239-258). A Coruña: Netbiblo.

Wilson, P.H., Spence, S.H. y Kavanagh, D. J. (1989) Cognitive behavioral interviewing for adult disorders: a practical handbook. Baltimore: The John Hopkins University Press.

Zander, L., Kreutzmann, M., West, S.G., Mettke, E. y Hannover, B. (2014). How school-based dancing classes change affective and collaborative networks of adolescents. Psychology of Sport and Exercise, 15, 418-428. doi.org/10.1016/j.psychsport.2014.04.004

Número de citas totales / Total references: 25 (100\%)

Número de citas propias de la revista / Journal's own references: $0(0 \%)$

Rev.int.med.cienc.act.fís.deporte - vol. 19 - número 75 - ISSN: 1577-0354 